\title{
Assessment of Inflammatory Markers in the Epidural Infusion of Bupivacaine-Fentanyl Compared to Morphine Bolus Injection in Gastrointestinal Cancer Surgeries
}

\author{
Seyed Jalal Madani ${ }^{1}$, Majid Saeedi ${ }^{*}$, Mohammad Saeed Gheasi ${ }^{1}$, Masoud Saghafinia ${ }^{1}$, Seyed Mohammad \\ Reza Amouzegar ${ }^{1}$, Ali Bahramifar ${ }^{1}$, Vahid Shahkarami'
}

${ }^{1}$ Trauma Research Center, Baqiyatallah University of Medical Sciences, Tehran, Iran

*Corresponding Author: Majid Saeedi, M.D., Trauma Research Center, Baqiyatallah University of Medical Sciences, Tehran, Iran. Tel: +98-21-88053766, Email: drmajidsaeedi@gmail.com

Received April 27, 2020; Accepted August 17, 2020; Online Published September 8, 2020

\begin{abstract}
Background: Gastrointestinal surgery is one of the procedures that result to produce inflammatory reactions and pain to patients. Administrate of high-safe analgesia in surgery is very essential, to reduce pain and improve inflammatory reactions.

Objectives: The present study compared inflammatory markers and pain relief with the epidural infusion of bupivacaine-fentanyl and intravenous morphine bolus in gastrointestinal cancer surgeries.

Methods: This randomized control clinical trial study was carried out from December 2018 to October 2020, on ASA I and II patients aged between 30-80 years who referred for gastrointestinal cancer surgery. Cases were randomly distributed into two groups. Group I received epidural catheter with bupivacaine (18 cases), and group II received fentanyl and morphine bolus injection (18 cases). On the first and second days post-operation, data on pain scores based on visual analogue scale (VAS) score, inflammatory markers (procalcitonin $[\mathrm{PCT}]$ and C-reactive protein [CRP]), platelet (PLT), white blood cells (WBCs), and erythrocyte sedimentation rate (ESR) was recorded.

Results: The mean age was $52.21 \pm 16.13$ years (41.6\% male, 58.4 female). The mean of ESR, CRP and PCT postoperative were significantly higher than preoperative $(P<0.05)$. There were no differences between the two groups regarding PLT, ESR, CRP, and PCT preoperative postoperative $(P>0.05)$. The mean of pain was less in the epidural group than in the morphine group postoperative $(P=0.02)$.

Conclusion: The results showed that epidural fentanyl-bupivacaine infusion was more effective in pain reduction than the intravenous infusion of morphine in cases undergoing gastrointestinal cancer operation. However, both analgesic approaches were similar in inflammatory functions.

Keywords: Inflammatory Biomarker, Pain, Epidural Analgesic, Morphine
\end{abstract}

\section{Background}

Tissue damage, pain, and anesthesia cause immunological alterations and metabolic and endocrine reactions in patients both perioperatively and postoperatively. ${ }^{1-3}$ These responses related to severity can have differing consequences, varying from the patient's susceptibility to infection, wound healing, systemic inflammatory response syndrome, and organ malfunction or failure. ${ }^{4-7}$

In addition to inflammation, increased inflammatory markers, and pain, following surgery, high doses of narcotics and non-opioid analgesics may be required. ${ }^{8,9}$ However, controversies remain about the effects of the types and amounts of anesthesia and postoperative analgesia on immunological reactions to major surgical procedures. ${ }^{10-12}$

Gastrointestinal surgery produces inflammatory reactions and pain for patients. The administration of highly safe analgesia in surgery is essential for reducing pain and improving inflammatory reactions. Inappropriate pain management may result in increased morbidity and, consequently, increased hospitalization and higher medical costs. ${ }^{8}$

Epidural analgesia is commonly used as an effective and safe strategy for pain relief in surgeries. ${ }^{8,9}$ It can reduce pain and effect earlier intubation times, better hemodynamics, and fewer respiratory complications. ${ }^{8}$

\section{Objectives}

The current study compared inflammatory markers and pain in epidural infusion of bupivacaine-fentanyl and morphine bolus injection in gastrointestinal cancer surgeries.

Copyright $\odot 2020$ The Author(s). This is an open-access article distributed under the terms of the Creative Commons Attribution License (http:// creativecommons.org/licenses/by/4.0), which permits unrestricted use, distribution, and reproduction in any medium, provided the original work is properly cited. 


\section{Methods}

This randomized control clinical trial was carried out from December 2018 to October 2020 and included 36 ASA I and II patients who referred for gastrointestinal cancer surgery. Participants ranged in age between 30-80 years.

Sample size, based on a medium effect size of 0.5 , a statistical power of $80 \%$, and a statistical significance of $0.05 \%$, was estimated to be 18 samples in each group using Gower-3.1.7 software and its sample size equation.

Exclusion criteria in this study were liver and kidney failure, uncontrolled endocrine or metabolic disorders, central nervous system disorders, active upper airway infection, patients treated with an alpha agonist or antagonist, pregnant women, history of any drug allergies, uncontrolled coagulation disorders, history of addiction, patients who experienced surgical complications during the study that required altering the level of anesthesia, or use of other hypnotic drugs.

Thirty-six cases were randomly allocated into two groups: group I: Epidural catheter with bupivacaine, group II: Fentanyl and morphine bolus injection. Blood samples were taken from both groups preoperatively and on the first and second days postoperative to measure inflammatory markers.

In the first group, anesthesia was performed by an epidural catheter specialist before standard anesthesia after complete monitoring and thorough sterilization prior to the induction of anesthesia in the sitting or lateral position and was fixed after skin canalization. Subsequently, $2 \mathrm{~mL}$ of the pump solution containing $20 \mathrm{cc}$ bupivacaine (1.5\%) and fentanyl $5 \mu / \mathrm{cc}$ and epinephrine $5 \mu / \mathrm{cc}$ were injected. The catheter was then connected to the $100 \mathrm{cc}$ pump at a speed of $6 \mathrm{cc} / \mathrm{h}$.

In the second group, intravenous bolus was injected with $0.1 \mathrm{mg} / \mathrm{kg}$ titration according to the patient's hemodynamics.

Both groups were injected with $2 \mathrm{~mL}$ of normal saline fluid and subjected to general anesthesia in a similar manner; premedication with $2 \mathrm{mg}$ midazolam and 100 $\mu \mathrm{g}$ fentanyl, induction with propofol $2 \mathrm{mg} / \mathrm{kg}$ titrated according to the patient's hemodynamics, atracurium $0.5 \mathrm{mg} / \mathrm{kg}$ relaxant, and propofol 50 to $150 \mu \mathrm{g} / \mathrm{min}$ were administered according to the hemodynamics of the patients.

The visual analogue scale (VAS) was used to assess pain severity at 1 and 2 days following cases entering the postanesthetic care unit. If VAS was greater than four, pethidine was administered intravenously at a dose of $0.5-1 \mathrm{mg} / \mathrm{kg}$.

On the first and second day post-operation, data was recorded regarding pain scores based on VAS score, inflammatory markers (procalcitonin [PCT] and C-reactive protein $[\mathrm{CRP}]$ ), platelets (PLT), white blood cells (WBCs), and erythrocyte sedimentation rate (ESR).

The data was analyzed using SPSS-20 software. Mann-Whitney $\mathrm{U}$ test and $t$ test were used according to the normality of data, which was examined using the Kolmogorov-Smirnov test. A $P$ value of less than 0.05 was considered statistically significant.

\section{Results}

Thirty-six patients were included in this study, $41.6 \%$ of whom were women. The overall average age was $52.21 \pm 16.13$ years, and no difference between the two groups regarding age, gender, and type of surgery was observed ( $P=0.48,0.56,0.31$, respectively) (Table 1 ).

Table 2 shows the comparison of inflammatory markers PLT, ESR, CRP, PCT, WBCs, and pain via VAS score at preoperative, 24 and 48 hours postoperative times. No differences were observed between the two groups regarding PLT, ESR, CRP, and PCT at preoperative, 24 and 48 hours postoperative times $(P>0.05$, Table 2$)$.

The means of ESR, CRP, and PCT were significantly higher at 24 and 48 hours postoperative than at preoperative $(P<0.05$, Table 2$)$.

There was no significant difference between the two groups in the preoperative mean of VAS (pain) $(P=0.89)$. The mean pain scores in the epidural group and morphine group were $2.65 \pm 1.56$ and $3.5 \pm 1.06$, respectively, at 24 hours postoperative. The mean pain score was lower in the epidural group than in the morphine group $(P=0.02)$. At 48 hours postoperative, mean pain scores in the epidural and morphine groups were $2.08 \pm 1.12$ and $2.8 \pm 0.98$, respectively. The mean of pain was lower in the epidural group than in the morphine group at 48 hours postoperative $(P=0.02)$. The rate of side effects in the epidural group was less than that in the morphine group $(P>0.05$, Table 2$)$. Pethidine consumption was lower in the epidural group than in the morphine group $(P>0.05$, Table 2$)$.

\section{Discussion}

This prospective study revealed that for pain relief, the postoperative analgesia properties of the epidural infusion of bupivacaine-fentanyl were better than those of morphine bolus injection in gastrointestinal cancer

Table 1. Comparison of Age, Gender, and Type of Surgery in the 2 Groups

\begin{tabular}{lcc}
\hline Characteristics & Epidural Group & Morphine Group \\
\hline Male, No. $(\%)$ & $10(55.5 \%)$ & $11(61.1 \%)$ \\
Female & $8(44.4 \%)$ & $7(38.8 \%)$ \\
Age, years, mean \pm SD & $53.92 \pm 16.21$ & $51.45 \pm 15.51$ \\
Types of surgery, No. $(\%)$ & & \\
Gastrostomy & $4(22.2 \%)$ & $6(33.3 \%)$ \\
Colectomy & $11(61.1 \%)$ & $9(50.0 \%)$ \\
Esophagectomy & $3(16.7 \%)$ & $3(16.7 \%)$ \\
\hline
\end{tabular}


Table 2. Comparison of Inflammatory Markers, Pain, Side Effects, and Pethidine Consumption at Preoperative, 24 and 48 Hours Postoperative Times

\begin{tabular}{|c|c|c|c|}
\hline Characteristics & Epidural Group & Morphine Group & $P$ Value \\
\hline PLT, count, preoperative & $190.6 \pm 63.64$ & $222.06 \pm 126.85$ & 0.67 \\
\hline 24 hours & $181.54 \pm 61.44$ & $232.62 \pm 93.48$ & 0.48 \\
\hline 48 hours & 172.51 .79 & $211.41 \pm 129.53$ & 0.56 \\
\hline$P$ value & 0.67 & 0.48 & \\
\hline $\mathrm{ESR}, \mathrm{mm} / \mathrm{h}$, preoperative & $21.00 \pm 12.24$ & $18.62 \pm 21.37$ & 0.55 \\
\hline 24 hours & $27.30 \pm 19.69$ & $25.85 \pm 17.55$ & 0.72 \\
\hline 48 hours & $45.50 \pm 22.66$ & $4.80 \pm 24.79$ & 0.64 \\
\hline$P$ value & 0.034 & 0.045 & \\
\hline CRP, mg/L, preoperative & $25.06 \pm 31.66$ & $24.60 \pm 27.76$ & 0.34 \\
\hline 24 hours & $53.57 \pm 26.81$ & $48.13 \pm 34.19$ & 0.16 \\
\hline 48 hours & $84.82 \pm 31.52$ & $77.79 \pm 31.99$ & 0.21 \\
\hline$P$ value & 0.021 & 0.038 & \\
\hline $\mathrm{PCT}, \mathrm{ng} / \mathrm{mL}$ preoperative & $0.05 \pm 0.00$ & $0.06 \pm 2.31$ & 0.87 \\
\hline 24 hours & $1.96 \pm 1.81$ & $1.70 \pm 8.20$ & 0.58 \\
\hline 48 hours & $2.98 \pm 3.69$ & $2.69 \pm 9.01$ & 0.45 \\
\hline$P$ value & 0.031 & 0.013 & \\
\hline WBCs, count, preoperative & $6909.09 \pm 2185.61$ & $7140.00 \pm 4257.22$ & 0.23 \\
\hline 24 hours & $9963.63 \pm 3814.77$ & $10500.00 \pm 5160.01$ & 0.02 \\
\hline 48 hours & $9809.9 \pm 3539.05$ & $12600.00 \pm 6313.79$ & 0.03 \\
\hline$P$ value & 0.041 & 0.039 & \\
\hline Pain, VAS, preoperative & $1.98 \pm 1.06$ & $1.89 \pm 1.08$ & 0.89 \\
\hline 24 hours & $2.65 \pm 1.56$ & $3.5 \pm 1.06$ & 0.02 \\
\hline 48 hours & $2.08 \pm 1.12$ & $2.8 \pm 0.98$ & 0.02 \\
\hline \multicolumn{4}{|l|}{ Complications } \\
\hline Vomiting, $\mathrm{N}$ & 4 & 9 & 0.017 \\
\hline Respiratory depression, $\mathrm{N}$ & 2 & 7 & 0.032 \\
\hline Non-return of bowel movements, $\mathrm{N}$ & 10 & 15 & 0.018 \\
\hline Pethidine consumption mg, $\mathrm{N}$ & 4 & 10 & 0.038 \\
\hline
\end{tabular}

surgeries. However, there was no difference between the two groups in inflammatory reactions, despite the increase in inflammatory markers in both groups postoperatively compared to baseline. Conflicts and controversies remain regarding the impact of the types of anesthesia and postoperative analgesia on the immunological reaction and inflammatory functions in postoperative patients. ${ }^{10-12}$

A systematic review and meta-analysis study by ConrickMartin et al demonstrated that the influence of spinal or epidural anesthesia was similar to that of general anesthesia on postoperative natural killer $\mathrm{T}$ lymphocyte reaction. ${ }^{10}$ A study of patients undergoing upper major abdominal surgery showed that general anesthesia combined with epidural anesthesia could not prevent immunosuppression produced by surgery. ${ }^{12}$

Vicente et $\mathrm{al}^{13}$ assessed biomarker markers in cases of hepatic metastatic disease who referred for hepatic resection using thoracic epidural analgesia and intravenous PCA. They showed that epidural analgesia could reduce inflammatory reactions and systemic immunosuppressive signaling in addition to promoting Th1 systemic signaling early after surgery. In this study, inflammatory biomarkers were significantly higher post-surgery than at baseline in the two groups; however, there was no difference between the two groups. The current study is in disagreement with the reported results. Due to limitations in laboratory kits for assessing specialized inflammatory markers, the current study could only assess PCT, CRP, platelet count, and ESR. Chloropoulou et $\mathrm{al}^{9}$ showed epidural anesthesia after epidural analgesia resulted in a weaker inflammatory reaction than spinal anesthesia after using intravenous morphine analgesia in cases surged with total knee arthroplasty. Consistent with the current results, they reported that CRPs were similar with the two analgesia methods. Moreover, Chloropoulou et $\mathrm{al}^{9}$ administered analgesia through spinal anesthesia followed by intravenous morphine analgesia in the control group; their control group and type of patients differed from those in the present study.

Ahlers et $\mathrm{al}^{11}$ showed that the intraoperative use of thoracic epidural catheter compared to intravenous morphine could decrease stress reaction and prohibited stress-induced perioperative damage of the inflammatory lymphocyte reaction in patients undergoing major abdominal surgery. They also showed that decreased perioperative stress is one of the most essential objectives. Another study reported that the sympathetic block induced by epidural anesthesia effected a profound suppression of hemodynamic and stress reactions to pediatric surgery and radical retropubic prostatectomies.

Several other studies have shown that the effectiveness of epidural anesthesia methods on postoperative pain and safety was better than intravenous anesthesia after major abdominal surgery, ${ }^{8,14}$ orthopedic surgery, ${ }^{9}$ and hepatic 


\section{Research Highlights}

\section{What Is Already Known?}

Epidural analgesia is a very effective and safe, commonlyused anesthesia strategy for pain reduction in surgeries. These anesthesia strategies are adequately utilized to reduce pain and provide earlier intubation times, better hemodynamics, and fewer respiratory complications.

\section{What This Study Adds?}

Epidural fentanyl-bupivacaine infusion was more effective in pain reduction than intravenous infusion of morphine in cases undergoing gastrointestinal cancer surgery.

resection. ${ }^{13}$ These results are consistent with the present study.

Teng et $\mathrm{al}^{15}$ demonstrated that patients who received epidural fentanyl bupivacaine PCA after a difficult surgery had greater pain relief than those receiving morphine PCA, either epidurally or intravenously, and experienced fewer side effects. In the present study also showed fentanyl is more preferred than morphine.

Consistent with the present study, Behera et $\mathrm{al}^{16}$ reported that epidural fentanyl and bupivacaine reduced pain and side effects more effectively than intravenous morphine after thoracic surgery.

Privado et $\mathrm{al}^{17}$ assessed epidural and intravenous fentanyl administered as postoperative analgesia in orthopedic surgeries. They reported that epidural fentanyl was more effective in relieving pain than intravenous fentanyl. Epidural analgesia can pass the remedy to sufficiently near the spinal cord so that the opioids can hinder pain release from afferent nerves to the central nervous system through communication with before and after synaptic opioid receptors in the dorsal horn. The epidural administration of PCA should produce more efficient analgesia than systemic treatment when the same quantity of an opioid is employed.

Epidural management of opioids is related to side effects including depression, pruritus, nausea, vomiting, delayed respiratory, urinary retention, and sedation. ${ }^{8}$ However, in the present study, no differences were observed between the two groups in the incident of any side effects.

\section{Conclusion}

The current results showed that epidural fentanylbupivacaine infusion was more effective in pain reduction than the intravenous infusion of morphine in cases undergoing gastrointestinal cancer operations. However, both analgesia approaches were similar in inflammatory functions.

\section{Authors' Contributions}

Conceptualization and data handling: SJM, MS; experiment design, data analysis: SJM, MS; materials:
SJM, MS; draft preparation: SJM, MS, MSG, MS, AB, VS; study consultation, writing and reviewing, research administration: SJM, MS, MSG, MS, AB, VS, SMRA.

\section{Conflict of Interest Disclosures}

All authors declare that they have no conflicts of interest in this study.

\section{Ethical Approval}

The current study was approved by the Ethics Committee of Baqiyatallah University of Medical Sciences (ethics code: ir.bmsu.rec.1397.037). Informed consent was confirmed and received from all cases.

\section{Acknowledgments}

The authors would like to thank th "Clinical Research Development Unit" of Baqiyatallah Hospital for their kind cooperation.

\section{References}

1. Munford RS, Pugin J. Normal responses to injury prevent systemic inflammation and can be immunosuppressive. Am J Respir Crit Care Med. 2001;163(2):316-321. doi:10.1164/ ajrccm.163.2.2007102.

2. Isitmangil G, Isitmangil T, Balkanli K, Cerrahoglu K, Kunter E. Detection of thoracotomy-induced alterations in cell- and humoral-mediated immune response. Eur J Cardiothorac Surg. 2002;21(3):497-501. doi:10.1016/s1010-7940(01)01165-4.

3. O'Sullivan ST, Lederer JA, Horgan AF, Chin DH, Mannick JA, Rodrick ML. Major injury leads to predominance of the T helper-2 lymphocyte phenotype and diminished interleukin-12 production associated with decreased resistance to infection. Ann Surg. 1995;222(4):482-490; discussion 490-482. doi:10.1097/00000658-199522240-00006.

4. Wolf AR. Effects of regional analgesia on stress responses to pediatric surgery. Paediatr Anaesth. 2012;22(1):19-24. doi:10.1111/j.1460-9592.2011.03714.x.

5. Hong JY, Yang SC, Yi J, Kil HK. Epidural ropivacaine and sufentanil and the perioperative stress response after a radical retropubic prostatectomy. Acta Anaesthesiol Scand. 2011;55(3):282-289. doi:10.1111/j.1399-6576.2010.02360.x.

6. Clementsen T, Reikerås O. Cytokine patterns after tourniquetinduced skeletal muscle ischaemia reperfusion in total knee replacement. Scand J Clin Lab Invest. 2008;68(2):154-159. doi:10.1080/00365510701528587.

7. Hughes SF, Hendricks BD, Edwards DR, Bastawrous SS, Roberts GE, Middleton JF. Mild episodes of tourniquet-induced forearm ischaemia-reperfusion injury results in leukocyte activation and changes in inflammatory and coagulation markers. J Inflamm (Lond). 2007;4:12. doi:10.1186/1476-9255-4-12.

8. Elzohry AAM, Abd-El-moniem Bakr M, Mostafa GM, Mohamad MF, Ahmed EH. Continuous perioperative thoracic epidural fentanyl-bupivacaine infusion vs. continuous perioperative fentanyl intravenous infusion in patients undergoing major upper abdominal cancer surgeries. J Pain Manage Med. 2008;4(1):132. doi:10.35248/2684-1320.18.4.132.

9. Chloropoulou P, latrou C, Vogiatzaki T, et al. Epidural anesthesia followed by epidural analgesia produces less inflammatory response than spinal anesthesia followed by intravenous morphine analgesia in patients with total knee arthroplasty. Med Sci Monit. 2013;19:73-80. doi:10.12659/msm.883749.

10. Conrick-Martin I, Kell MR, Buggy DJ. Meta-analysis of the effect of central neuraxial regional anesthesia compared with general anesthesia on postoperative natural killer T lymphocyte 
function. J Clin Anesth. 2012;24(1):3-7. doi:10.1016/j. jclinane.2011.09.001.

11. Ahlers $\mathrm{O}$, Nachtigall I, Lenze J, et al. Intraoperative thoracic epidural anaesthesia attenuates stress-induced immunosuppression in patients undergoing major abdominal surgery. Br J Anaesth. 2008;101(6):781-787. doi:10.1093/bja/ aen287.

12. Kawasaki T, Ogata M, Kawasaki C, Okamoto K, Sata T. Effects of epidural anaesthesia on surgical stress-induced immunosuppression during upper abdominal surgery. $\mathrm{Br}$ J Anaesth. 2007;98(2):196-203. doi:10.1093/bja/ael334.

13. Vicente $D$, Patino $M$, Marcus $R$, et al. Impact of epidural analgesia on the systemic biomarker response after hepatic resection. Oncotarget. 2019;10(5):584-594. doi:10.18632/ oncotarget.26549.

14. Kawasaki T, Ogata M, Kawasaki C, Okamoto K, Sata T.
Effects of epidural anaesthesia on surgical stress-induced immunosuppression during upper abdominal surgery. $\mathrm{Br} J$ Anaesth. 2007;98(2):196-203. doi:10.1093/bja/ael334.

15. Teng YH, Hu JS, Tsai SK, Liew C, Lui PW. Efficacy and adverse effects of patient-controlled epidural or intravenous analgesia after major surgery. Chang Gung Med J. 2004;27(12):877-886.

16. Behera BK, Puri GD, Ghai B. Patient-controlled epidural analgesia with fentanyl and bupivacaine provides better analgesia than intravenous morphine patient-controlled analgesia for early thoracotomy pain. J Postgrad Med. 2008;54(2):86-90. doi:10.4103/0022-3859.40772.

17. Privado MS, Issy AM, Lanchote VL, Garcia JB, Sakata RK. Epidural versus intravenous fentanyl for postoperative analgesia following orthopedic surgery: randomized controlled trial. Sao Paulo Med J. 2010;128(1):5-9. doi:10.1590/s151631802010000100002 . 